\title{
Nuclear factor I/B mediates epithelial-mesenchymal transition in human melanoma cells through ZEB1
}

\author{
RUIMIN CHENG, SHENG GAO, WEI HU, YAMEI LIU and YUCHUN CAO \\ Department of Dermatology, Tongji Hospital, The Tongji Medical College of Huazhong \\ University of Science and Technology, Wuhan, Hubei 430030, P.R. China
}

Received January 25, 2019; Accepted September 10, 2020

DOI: $10.3892 / \mathrm{ol} .2020 .12342$

\begin{abstract}
The relationship between nuclear factor I/B (NFIB) and cancer attracts growing research interest. NFIB has diverse and specific roles in tumor progression and invasion. However, the potential effects and functions of this transcription factor in melanoma remain unclear. The present study sought to determine the distinguishing properties of NFIB in melanoma cells. Immunohistochemical examination of the tissues of 15 patients with melanoma indicated that the expression of NFIB was high in melanoma specimens, compared with the benign nevus and normal skin specimens. In addition, the relationship between high NFIB expression and low overall survival rate was assessed. Functional studies demonstrated that NFIB enhanced the malignancy of melanoma, including proliferation, migration and invasion. In addition, NFIB silencing in A375 and A875 cell lines inhibited the process of epithelial-mesenchymal transition (EMT), upregulated E-cadherin and zona occludens-1, but suppressed N-cadherin and vimentin expression. These findings may suggest a new function of NFIB in promoting the migration and invasion of melanoma cells. Therefore, the present study further evaluated the association between NFIB and zinc finger protein E-box binding homeobox-1 (ZEB1) in melanoma. Mechanistic experiments revealed that NFIB exerted its roles during EMT by regulating ZEB1. Overall, the present data indicates that NFIB promotes the malignancy of melanoma, particularly EMT, by modulating the ZEB1 axis, such as ZEB2, ATM and CHK1, which may represent a potential molecular therapeutic target in melanoma.
\end{abstract}

Correspondence to: Dr Yuchun Cao, Department of Dermatology, Tongji Hospital, The Tongji Medical College of Huazhong University of Science and Technology, 1095 Jiefang Avenue, Wuhan, Hubei 430030, P.R. China

E-mail: 963808187@qq.com

Key words: melanoma, nuclear factor I/B, epithelial-mesenchymal transition, zinc finger protein E-Box binding homeobox 1, metastasis

\section{Introduction}

Malignant melanoma, which arises from melanocytes, is lethal and common in the global population, ranking 5th for the incidence of malignant tumors in males, and 6th in females for mortality rate (1). Furthermore, its mortality seriously threatens human health and imparts a grave economic burden (2,3). Although surgical resection of malignant tissue is recognized as the most effective therapy in the early stages of melanoma, the 5-year survival rate in metastatic melanoma is only $15 \%$, with patients experiencing a very poor prognosis $(4,5)$. Furthermore, melanoma is characterized by high rates of recurrence with high mortality, often diagnosed in the last stages and resistant to current therapeutic approaches $(6,7)$. Therefore, further studies are needed to identify novel biomarkers for malignant melanoma and to develop a deeper understanding of the mechanisms that lead to melanoma progression.

Epithelial-mesenchymal transition (EMT) is an indispensable biological process that is closely connected to embryogenesis and the first stage of wound healing (8). Recently, it has become widely acknowledged that the mechanism of EMT is more complex in melanoma. Indeed, abnormal activation of the EMT pathway alters the microenvironment by which epithelial tumor cells that initially undergo EMT are able to revert to epithelial phenotype by mesenchymal-epithelial transition at the distant site, therefore tumor cells penetrate the basement membrane and migrate (9). A variety of transcription factors act as molecular switches that directly regulate the occurrence of the EMT process in melanoma $(10,11)$. Among these, zinc finger protein E-box binding homeobox (ZEB) proteins, especially ZEB1 and -2 , participate in the initiation of EMT in melanoma by downregulating the expression in microphthalmia-associated transcription factor (MITF) (12).

Nuclear factor I (NFI) is a type of transcription factor family, which is widely found in mammals and also known as CCAAT box-binding transcription factor (CTF). This family is characterized by a highly conserved N-terminal DNA-binding region, and divided into four subtypes (A, B, $\mathrm{C}$ and $\mathrm{X}$ ) based on the variable $\mathrm{C}$-terminus region $(13,14)$. NFIs are known to be involved in the regulation of DNA replication and gene expression and to promote cell proliferation and differentiation during the embryonic development (15-17). 
In addition, NFIs are abnormally expressed in various tumors and has a complex and diverse function in some tumors (18). For instance, human NFI type-B (NFIB) serves a crucial role in different aspects of tumor development, acting as an oncogene or tumor suppressor in different types of tumor and participating in tumor-associated fusion gene formation (19-21). Previous studies on the role of NFIB in colorectal cancer (CRC) have indicated that NFIB triggers EMT of CRC cells through upregulation of SNAI1 expression (22). Moreover, NFIB seems to mediate the conversion between the two transcription factors, POU class 3 homeobox 2 and MITF, through the upregulation of enhancer of zeste homolog 2, which increases expression of MITF and decreases expression of BRN2, thus driving the invasive phenotype in the melanoma (23). However, few studies have been conducted on the specific mechanisms involved in melanoma metastasis based on NFIB. Thus, the present study investigated the impact of NFIB on EMT in A375 and A875 cell lines. Given that ZEB1 has two possible binding sites for the NFIB promoter (24), it was hypothesized that there was an association between ZEB1 and NFIB. The findings of this study may provide insight into the metastasis of malignant melanoma, with particular emphasis on EMT.

\section{Materials and methods}

Clinical specimens. To detect the expression of NFIB by immunohistochemistry (IHC), 15 melanoma samples, 15 benign nevus samples and 10 normal skin samples were collected between December 2017 and January 2018 from different patients. The total number of patients enrolled for the study was 40 . Clinical samples of melanoma and nevus in this study were provided and pathologically diagnosed by the Department of Dermatology, Tongji Hospital affiliated with Huazhong University of Science and Technology, while normal skin samples were obtained from the Department of Urology, Tongji Hospital affiliated with Huazhong University of Science and Technology. These tissue specimens were fixed in $10 \%$ formalin at room temperature after resection. After $24 \mathrm{~h}$ of fixation, the samples were then embedded in paraffin for IHC. All the participants were newly diagnosed and had not received any comprehensive antitumor treatment before the surgical section. They were informed of their rights and signed an informed consent form. The present study was approved by the Ethics Review Committee at Tongji Hospital affiliated with Huazhong University of Science and Technology.

IHC analysis. The expressions of NFIB protein in the samples of melanoma, nevus and normal skin were detected by IHC. All three kinds of specimens were cut into uniform sections of $3-\mu \mathrm{m}$ thickness. The samples were treated with $10 \%$ polylysine, then fixed on glass slides using melted paraffin at $65^{\circ} \mathrm{C}$ for 5 min and baked in an oven at $65^{\circ} \mathrm{C}$ overnight. The next day, the prepared sections were hydrated by dewaxing with graded alcohol and xylene at room temperature. Thereafter, antigen retrieval was carried out using $600 \mathrm{ml} 10 \mathrm{mM}$ sodium citrate (pH 6.0) heated in a microwave oven at $100^{\circ} \mathrm{C}$ for $6-8 \mathrm{~min}$ and then cooled at room temperature. Several drops of hydrogen peroxide were then added for incubation at room temperature for $15 \mathrm{~min}$ to neutralize the excess oxygen radicals and reduce the background. Then, a primary antibody specific for NFIB (cat. no. ab186738; 1:100 dilution; Abcam) was added to the sections at $4{ }^{\circ} \mathrm{C}$ overnight, followed by incubation with a secondary antibody conjugated with streptavidin-HRP at room temperature for $30 \mathrm{~min}$. A freshly prepared DAB coloring solution was used to enhance coloration with hematoxylin re-dyeing, $1 \%$ hydrochloric acid ethanol differentiation, trypan blue pan-blue and gradient alcohol dehydration. Subsequently, the relative intensity of NFIB expression was evaluated using Image-Pro Plus software (IPP version 6.0; Media Cybernetics, Inc.).

Bioinformatics analysis. The present study analyzed from TCGA website (https://xenabrowser.net/heatmap/) and the Oncomine Cancer Microarray database (https://www.oncomine.org/resource/login. html) to compare the expression levels of NFIB mRNA in 227 samples, including 92 melanoma, 85 benign nevus and 50 normal skin specimens. In each dataset, the median value of NFIB expression was used to divide the samples into an NFIB high-expression group and an NFIB low-expression group. The analysis was performed using GraphPad Prism 5.0 and log-rank tests (GraphPad Software, Inc.).

Cell lines and culture condition. The human melanoma cell lines A375, A875 and SK-MEL-1, and the normal HaCaT keratinocyte cell line were purchased from the China Center for Type Culture Collection. HaCaT cells were authenticated by STR. All cell lines were resuspended in Dulbecco's Modified Eagle Medium (DMEM; Gibco; Thermo Fisher Scientific, Inc.) supplemented with $10 \%$ fetal bovine serum (FBS; Gibco; Thermo Fisher Scientific, Inc.) and incubated at $37^{\circ} \mathrm{C}$ with $5 \% \mathrm{CO}_{2}$. The cell culture medium was replaced or the cells were sub-cultured, as appropriate, every 1-2 days.

Cell transfection. A375 and A875 cells were uniformly seeded into 6-well plates at $4 \times 10^{5}$ cells/well and incubated overnight. The cells were resuspended in Opti-MEM (Thermo Fisher Scientific, Inc) then transfected with small interfering (si) RNA (si-NFIB, 5'-AGGAUACUCUGAAGAACUAUU-3'; Guangzhou RiboBio Co., Ltd.) to silence NFIB expression at $50 \mathrm{nM} /$ well. The transfected cells were screened using puromycin $(5.0 \mu \mathrm{g} / \mathrm{ml})$. The recombinant plasmid pcDNA.3.1-NFIB (Guangzhou RiboBio Co., Ltd.) was transfected into A375 and A875 cells to induce NFIB overexpression at $0.2 \mu \mathrm{g} /$ well. si-negative control (NC; 5'-TTCTCCGAACGTGTCACGTdT dT-3') and empty vectors were used as controls. Silencing and overexpression efficiency were examined by western blot and reverse transcription-quantitative PCR (RT-qPCR). After 10 days of lentivirus transfection or $72 \mathrm{~h}$ after siRNA transfection, western blot and RT-PCR analyses were used to detect the expression of target genes. Plasmids pcDNA-NFIB were transfected into $293 \mathrm{~T}$ cells for luciferase reporter assays.

Establishment of an EMT model of melanoma cells. The A375 and A875 cells were uniformly seeded into a 6-well plate at a density of $4 \times 10^{5}$ cells/well, and cultured to $\sim 75 \%$ confluence. Cells were then treated with $5 \mathrm{ng} / \mathrm{ml}$ TGF- $\beta 1$. TGF- $\beta 1$ (PeproTech, Inc.) was used to stimulate EMT. Untreated cells were used as a control. A phase-contrast microscope (Model 
CKX41; magnification, x100; Olympus Corporation) was used to examine cell morphological changes after $24 \mathrm{~h}$.

$R T-q P C R$. Total RNA was extracted from cells $48 \mathrm{~h}$ following transfection using TRIzol ${ }^{\circledR}$ solution following the manufacturer's instructions (Thermo Fisher Scientific, Inc.). An ultraviolet spectrophotometer with a wavelength between 260 and $280 \mathrm{~nm}$ was used to measure the RNA concentration. Total RNA was reverse transcribed to cDNA with RT Master Mix (Takara Bio, Inc.) according to the manufacturer's instructions. RT-PCR was performed with SYBR Master Mix (Takara Bio, Inc.) using the StepOne-Plus system (Thermo Fisher Scientific, Inc.). RT-PCR was performed with 40 cycles under the following conditions: Denaturation at $95^{\circ} \mathrm{C}$ for $30 \mathrm{sec}$, annealing at $60^{\circ} \mathrm{C}$ for $1 \mathrm{~min}$ and extension at $95^{\circ} \mathrm{C}$ for $5 \mathrm{sec}$. Each experiment was conducted three times. The specific primer sequences for evaluating the NFIB expression in melanoma cell lines were as follows: i) NFIB forward, 5'-AAAAAGCATGAGAAGCGA ATGTC-3'; ii) NFIB reverse, 5'-ACTCCTGGCGAATATCTT TGC-3'; iii) GAPDH forward, 5'-ACAACTTTGGTATCG TGGAAGG-3'; and iv) GAPDH reverse, 5'-GCCATCACG CCACAGTTTC-3'. GAPDH served as an endogenous control to normalize NFIB expression in each sample. The relative expression of NFIB was calculated using the comparative $\mathrm{Cq}$ $\left(2^{-\Delta \Delta \mathrm{Cq}}\right)$ method (25).

Cell proliferation. The Cell Counting Kit-8 (CCK-8; Beijing Baisi Biological Technology Co., Ltd.) was used to evaluate the rate of cell proliferation. In brief, A375 and A875 cells were uniformly seeded into three 96-well plates at a density of $1 \times 10^{3}$ cells/well, in culture medium volume $100 \mu \mathrm{l} /$ well. $10 \mu \mathrm{l}$ CCK-8 solution was added into the media. The absorbance of cells was assessed at a fixed time every day, and the data were continuously detected by a microplate reader (Thermo Fisher Scientific, Inc.). The optical density of cells was measured at a $450 \mathrm{~nm}$ wavelength. The cells were incubated for 24 , 48 and $72 \mathrm{~h}$ at $37^{\circ} \mathrm{C}$ with $5 \% \mathrm{CO}_{2}$.

Colony formation assay. Melanoma cells in the logarithmic growth period were seeded in a 6-well plate at a density of $5 \times 10$ cells/well containing DMEM medium with $10 \%$ FBS and incubated at $37^{\circ} \mathrm{C}$ in a humidified incubator with $5 \% \mathrm{CO}_{2}$. When the colonies were visible to the naked eye, the culture was stopped immediately and then fixed with $4 \%$ paraformaldehyde (Google Biotechnology Co., Ltd.) and stained by $0.1 \%$ crystal violet (Google Biotechnology Co., Ltd.). Finally, the number of colonies ( $>50$ cells) were analyzed under an inverted microscope (Olympus Corporation), and the colony number and colony formation rates were calculated. Clone formation rate $=$ (number of clones/number of inoculated cells) $\mathrm{x} 100 \%$.

Wound healing assay. To assess the effects of NFIB on cell migration, $5 \times 10^{4} \mathrm{~A} 375$ and A875 cells were seeded during the logarithmic growth period in the 6-well plate to ensure that the cells would reach $90-100 \%$ confluency the next day. Three parallel lines were drawn on the back of the 6-well plate with a marker pen, and a $100-\mu 1$ pipette tip was selected to draw a line on the bottom of the cell of the 6-well plate. After this, the cells were washed with sterile PBS repeatedly 3-5 times to remove floating cell debris. The cells were then cultured in serum-free medium for 24 and $48 \mathrm{~h}$. Migration between scratches was observed under an inverted microscope under x100 magnification and kept at the same position and time under the mark.

Cell migration and invasion assay. Migration and invasion were evaluated in 24-well Transwell chambers (Corning Inc.) in the presence or absence of Matrigel ${ }^{\mathrm{TM}} \mathrm{ECM}$ (Corning Inc.) coating. After transfection for $48 \mathrm{~h}, \mathrm{~A} 375\left(5 \times 10^{4}\right.$ cells/chamber $)$ and A875 (1x10 cells/chamber) cells resuspended in $200 \mu \mathrm{l}$ of serum-free DMEM medium were seeded into the upper chamber. The lower chamber received $600 \mu 1$ of DMEM medium mixed with $10 \%$ FBS. Then, the cells were incubated for an additional $24 \mathrm{~h}$ for migration assays or $48 \mathrm{~h}$ for invasion assays at $37^{\circ} \mathrm{C}$ with $5 \% \mathrm{CO}_{2}$. Finally, the cells in the upper chamber were wiped off with a cotton bud, and the penetrated cells underneath the chamber were fixed with $4 \%$ paraformaldehyde and stained with $0.1 \%$ crystal violet staining solution (both at room temperature for $30 \mathrm{~min}$ ). The cells were counted in five randomly selected fields for each membrane under an inverted microscope and photographed at x100 magnification.

Western blot analysis. Total protein was extracted using RIPA buffer (Thermo Fisher Scientific, Inc.) supplemented with protease inhibitor cocktail and 1\% PMSF (Roche Diagnostics). The protein was quantified using a BCA Protein Assay Reagent kit (Thermo Fisher Scientific, Inc.) and were collected and mixed with loading buffer. Equal amounts of protein (40 $\mu \mathrm{g}$ ) from each sample were separated by $10 \%$ SDS-PAGE and transferred to PVDF membranes (EMD Millipore). The membranes were blocked in 5\% milk in TBST buffer for $2 \mathrm{~h}$ at room temperature, then incubated with primary antibodies at the recommended dilution overnight at $4^{\circ} \mathrm{C}$. After binding with secondary antibody conjugated HRP at room temperature for $1 \mathrm{~h}$, Image J (National Institutes of Health) was used to measure the band density. GAPDH acted as an internal reference, and each sample was analyzed three times. NFIB $(1: 1,000$; cat. no. ab80835) and GAPDH (1:1,000; cat. no. ab9485) antibodies were purchased from Abcam. Vimentin (1:1,000; cat. no. 10366-1-AP) were purchased from ProteinTech Group, Inc. E-cadherin (1:1,000; cat. no. sc-71007) and N-cadherin (1:1,000; cat. no. sc-71002) were purchased from Santa Cruz Biotechnolgy, Inc. Secondary horseradish peroxidase-goat anti-rabbit antibodies (1:1,000; cat. no. 10285-1-AP) were purchased from ProteinTech Group, Inc.

Statistical analysis. All data are presented as the mean \pm standard deviations (SD) from three independent experiments. All statistical analyses were performed using GraphPad Prism (version 6.0; GraphPad Software, Inc.). Unpaired Student's t-test was employed to compare the difference between two groups. One-way ANOVA followed by the Least Significant Difference was used for multigroup comparisons. The log-rank test and Kaplan-Meier survival curves were used to analyze the association between NFIB expression and overall survival. Statistical analyses were performed using GraphPad Prism 5.0 (GraphPad Software, Inc.). $\mathrm{P}<0.05$ was considered to indicate a statistically significant difference. 

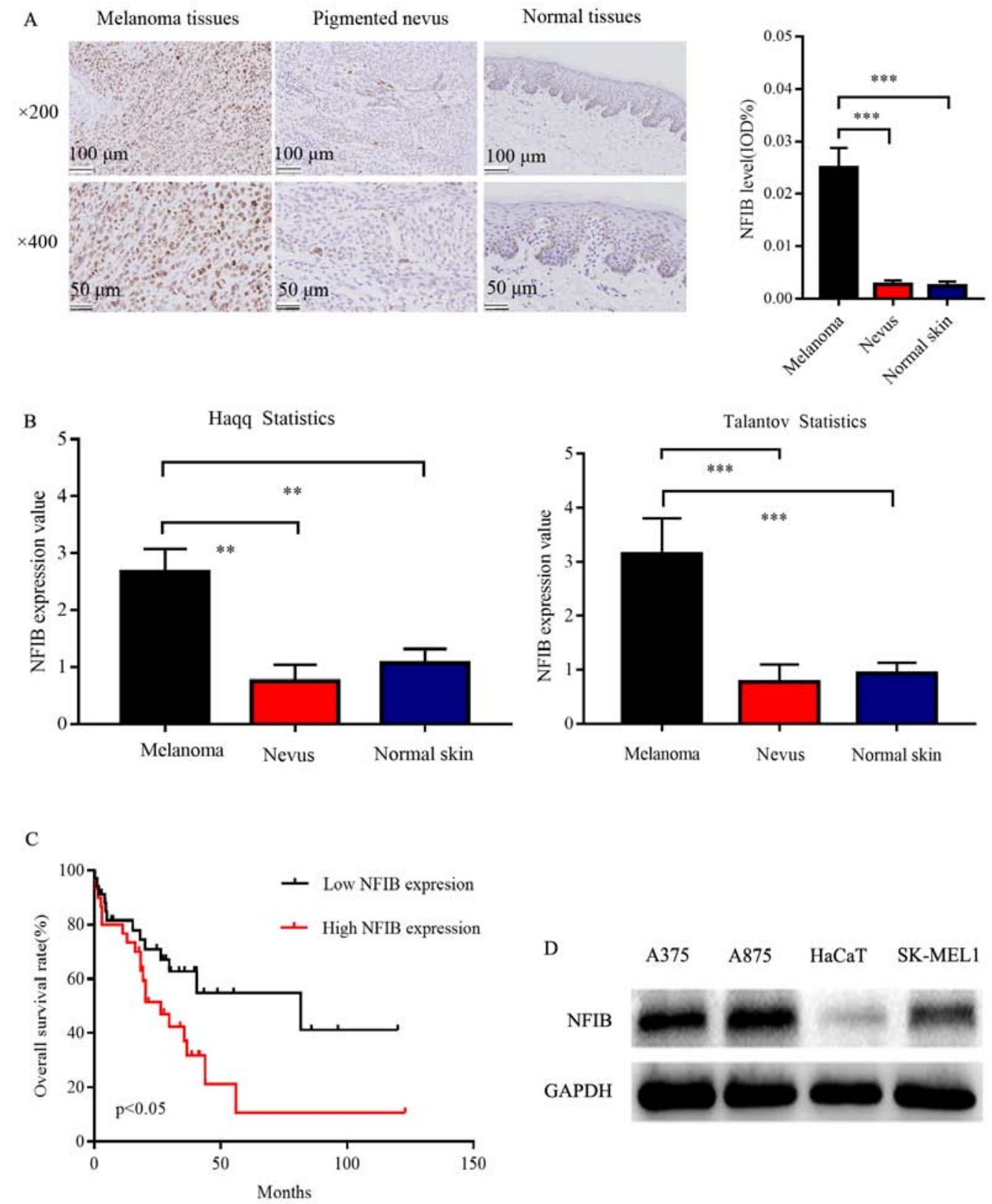

Figure 1. NFIB is highly expressed in human malignant melanoma tissues and associated with poor prognosis. (A) Immunohistochemical staining of NFIB in melanoma tissues, paired with benign nevus and normal skin. $\mathrm{P}<0.001$, one-way ANOVA test. (B) NFIB mRNA expression in melanoma, nevus and normal skin in two Oncomine datasets. ${ }^{* *} \mathrm{P}<0.01,{ }^{* * *} \mathrm{P}<0.001$; one-way ANOVA test. (C) Based on the mean NFIB expression set by the TCGA database website, patients with melanoma were divided into high and low NFIB expression groups. Kaplan-Meier survival analysis and log-rank test were used to evaluate the effect of NFIB expression on overall survival. (D) NFIB protein expression in the A375, A875 and SK-MEL1 human melanoma cell lines and the normal human HaCaT keratinocytes. NFIB, nuclear factor I/B.

\section{Results}

NFIB is relatively high in human malignant melanoma and associated with poor prognosis. The expression and molecular mechanism of NIFB have not been clearly reported in melanoma previously. Initially, the present study analyzed the localization of NFIB in clinical samples. NFIB expression levels were examined by IHC in 15 malignant melanoma samples, 15 benign nevus samples and in 10 normal skin non-matched samples. In total, 12 malignant melanoma samples were positive for NFIB expression, five benign nevus samples were positive for NFIB expression, no normal skin sample was positive for NFIB expression. Notably, NFIB was widely distributed in melanoma samples with a cytonuclear staining pattern (data not shown). A significantly higher expression of NFIB was observed in the melanoma specimens, compared with benign pigmented nevus and normal human skin (Fig. 1A). Furthermore, mRNA expression data from the Haqq and Talantov Oncomine datasets supported these findings (Fig. 1B).

The Oncomine datasets were also used to explore the relationship between NFIB expression levels and overall survival. The log-rank test and Kaplan-Meier survival analysis indicated that high NFIB expression was associated with lower overall survival rate, relative to the NFIB low-expression group (Fig. 1C). This indicated that NFIB may serve as a poor 

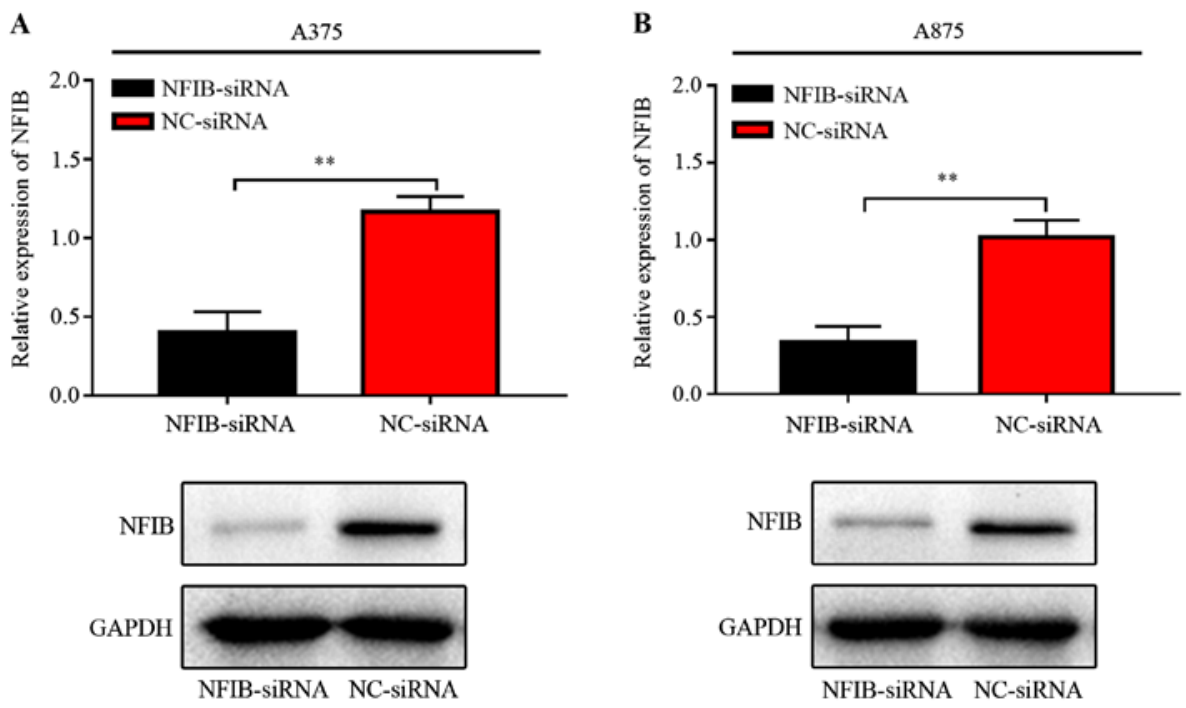

Figure 2. Detection of knockdown efficiency in melanoma cells transfected with NFIB-siRNA. (A) A375 and (B) A875 cell lines were transfected with NFIB-siRNA or NC-siRNA, and NFIB expression was measured using reverse transcription-quantitative PCR and western blot analysis. ${ }^{* *} \mathrm{P}<0.01 \mathrm{NFIB}$, nuclear factor I/B; siRNA, small interfering RNA; NC, negative control.
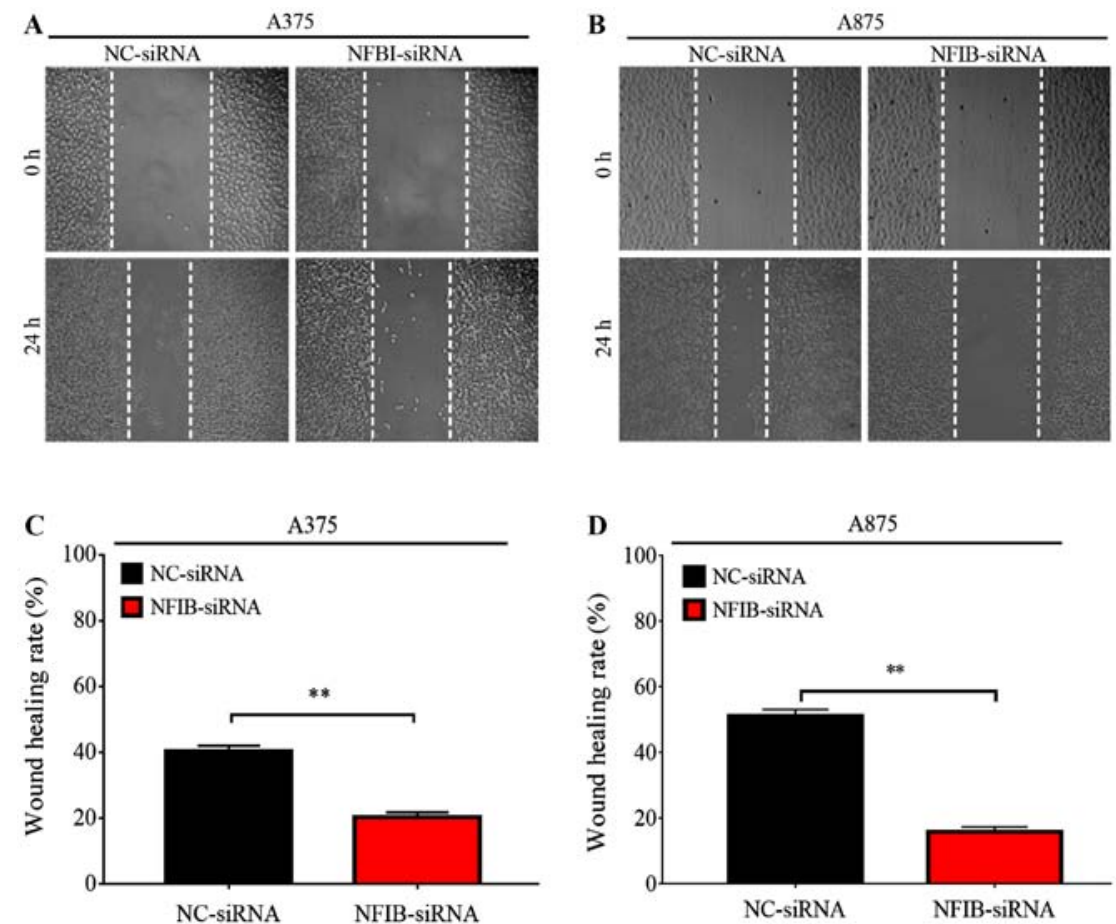

Figure 3. NFIB expression promotes cell migration. Wound healing assays were used to evaluate the capacity of cell migration following transfection with NFIB-siRNA or NC-siRNA in both (A) A375 and (B) A875 cells. Statistical analysis of wound healing rates in (C) A375 and (D) A875 cells. ${ }^{* *} \mathrm{P}<0.01$. NFIB, nuclear factor I/B; siRNA, small interfering RNA; NC, negative control. Magnification, x100.

prognostic indicator for melanoma and could be associated with the aggressiveness of melanoma cells.

In addition, western blotting suggested that NFIB expression levels were also elevated in the A375, A875 and SK-MEL1 melanoma cell lines, compared with the normal human keratinocyte $\mathrm{HaCaT}$ cell line (Fig. 1D).

NFIB expression promotes migration and invasion in melanoma cell lines. To further investigate the role of NFIB on malignancy in melanoma, NFIB expression was silenced in
A375 and A875 cells using transfection with NFIB-siRNA. Detection of knockdown efficiency was done by RT-qPCR and western blot analysis. NFIB expression was notably dropped in melanoma cell lines using transfection with NFIB-siRNA compared with NC-siRNA (Fig. 2). Subsequently, the role of NFIB on migration and invasion in melanoma cell lines was evaluated in wound healing and Transwell assays. Transfection with NFIB-siRNA significantly reduced the migratory capacity of both A375 and A875 cells, compared with NC-siRNA $(\mathrm{P}<0.01$; Figs. 3 and 4). In addition siRNA-mediated NFIB 

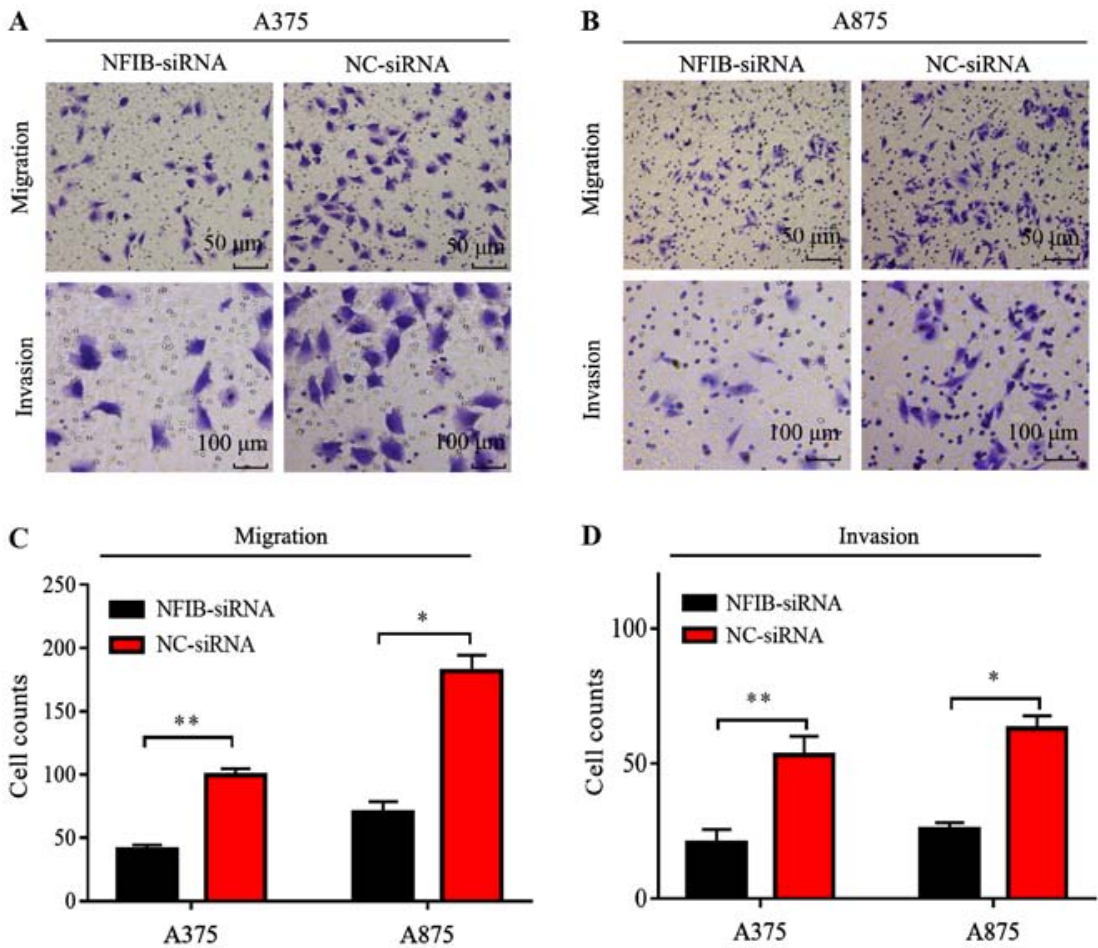

Figure 4. Expression of NFIB promotes cell migration and invasion. Transwell assays were used to evaluate cell migration and invasion following transfection with NFIB-siRNA or NC-siRNA in (A) A375 and (B) A875 cells. Statistical analysis of (C) migration and (D) invasion assays. ${ }^{*} \mathrm{P}<0.05,{ }^{* *} \mathrm{P}<0.01 . \mathrm{NFIB}$, nuclear factor I/B; siRNA, small interfering RNA; NC, negative control.

A

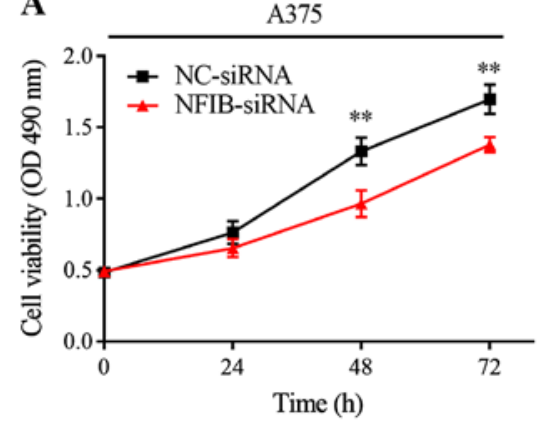

B

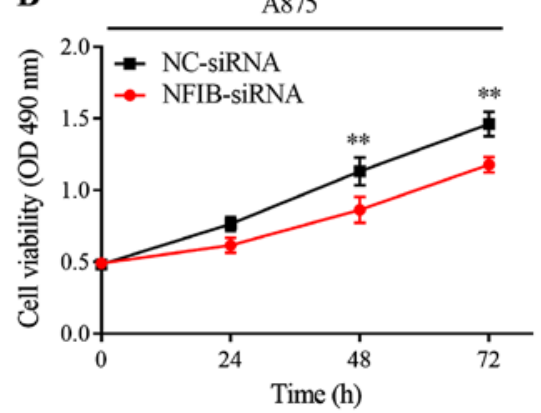

Figure 5. Expression of NFIB promotes proliferation. Cell Counting Kit-8 assays were used to evaluate cell proliferation. In NFIB-siRNA-transfected (A) A375 and (B) A875 cells, the proliferative ability was reduced, compared with NC-siRNA., ${ }^{* *} \mathrm{P}<0.01$. NFIB, nuclear factor I/B; siRNA, small interfering RNA; NC, negative control; OD, optical density.

silencing also significantly reduced the invasion capacity of both A375 and A875, relative to the NC-siRNA (Fig. 4).

NFIB promotes melanoma cell proliferation and colony formation. Considering that NFIB is highly expressed in human malignant melanoma, the present study sought to investigate whether NFIB affects colony formation and proliferation of melanoma cells. In CCK-8 assays, proliferative ability was significantly reduced in NFIB-silenced melanoma cell lines relative to their respective NC-siRNA controls (Fig. 5A and B). Interestingly, the inhibition effect on the proliferation of melanoma cells began to appear in the first $24 \mathrm{~h}$, and peaked between 24 and $48 \mathrm{~h}$, whereas this effect disappeared after $48 \mathrm{~h}$. Furthermore, the colony formation rate of cells transfected with NFIB-siRNA was significantly lower than that of the NC-siRNA group in A375 and A875 cells (Fig. 6B and D).
Furthermore, compared with the NC-siRNA, cells transfected with NFIB-siRNA also exhibited restricted colonies in both size and numbers (Fig. 6A and C). Consequently, these data suggested that NFIB may enhance the malignancy of melanoma cells, by enhancing the proliferative ability.

NFIB expression promotes EMT in melanoma cell lines. A large number of studies have suggested that EMT has an important role in malignant tumors, focusing on malignant behaviors, such as migration and invasion. During the progression of EMT, E-cadherin is known to be downregulated and $\mathrm{N}$-cadherin is upregulated, which reduces the polarity of epithelial cells and weakens the connection with the basement membrane to obtain higher invasion and migration capacities (26). Thus, the present study examined whether NFIB had an effect on melanoma EMT. Morphological changes of the 
A

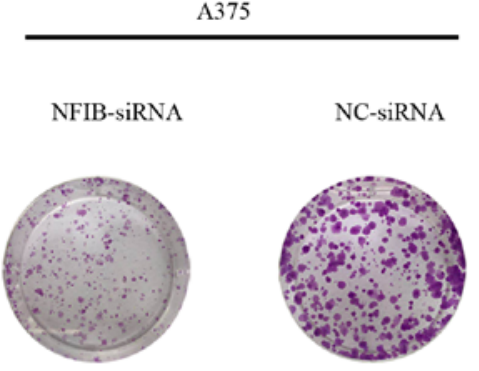

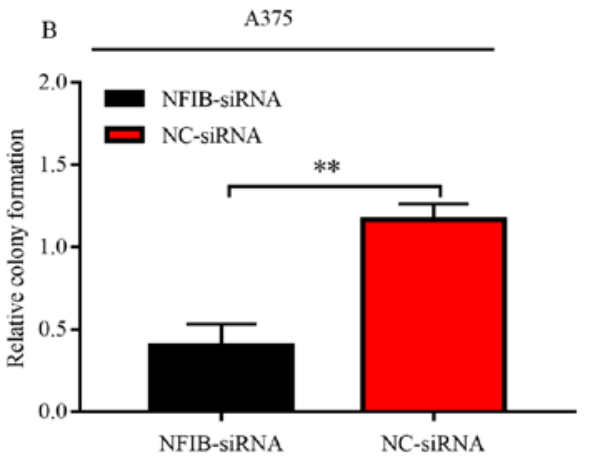

$\mathrm{C}$

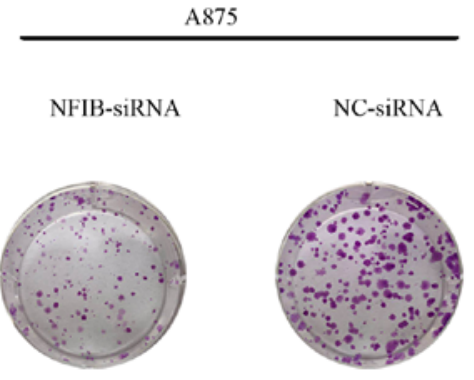

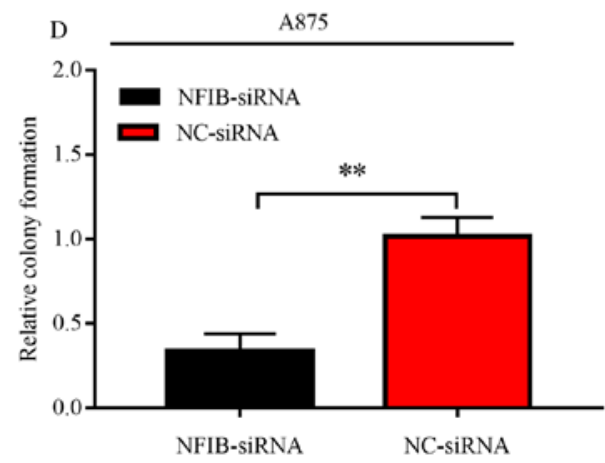

Figure 6. Expression of NFIB promotes proliferation colony formation. Colony formation assays were used to evaluate cell migration and colony formation in NFIB-siRNA or NC-siRNA-transfected (A) A375 and (C) A875 cells. Statistical analysis of colony formation assays in both (B) A375 and (D) A875 cells, ${ }^{* *} \mathrm{P}<0.01$. NFIB, nuclear factor I/B; siRNA, small interfering RNA; NC, negative control.

A
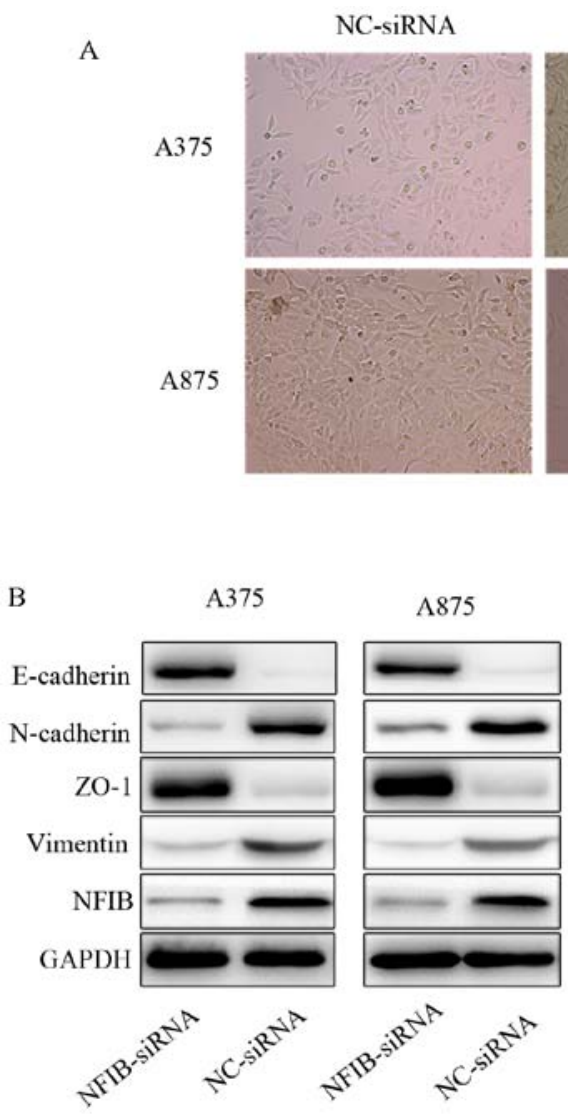

NC-siRNA+TGF- $\beta 1 \quad$ NFIB-siRNA+TGF- $\beta 1$
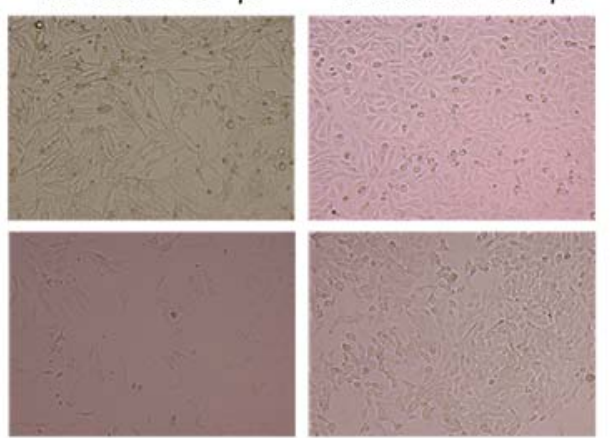

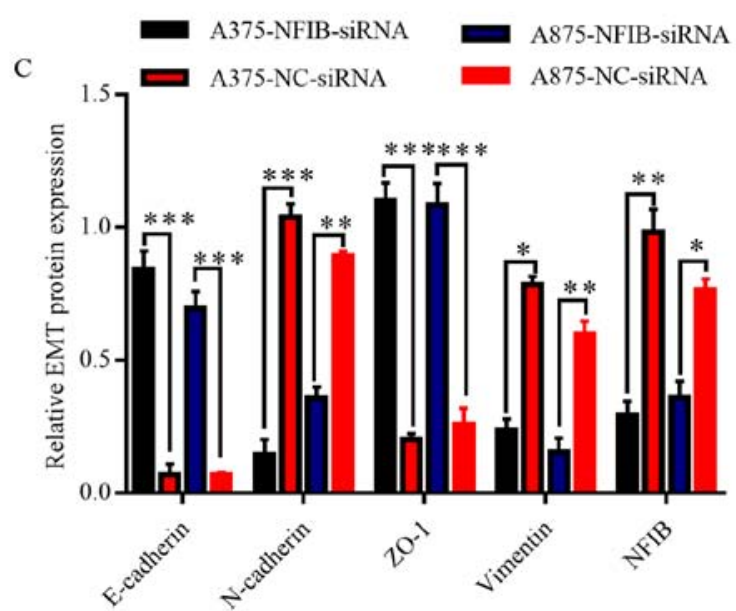

Figure 7. NFIB promotes invasion and proliferation of A375 and A875 cells by facilitating EMT in vitro. (A) Inverted phase-contrast microscopy of melanoma cells following transfection with NFIB-siRNA or NC-siRNA and TGF- $\beta 1$ treatment. (B) Western blots and (C) semi-quantitative analysis of epithelial phenotype markers E-cadherin, N-cadherin, ZO-1, vimentin, as well as NFIB following transfection. ${ }^{*} \mathrm{P}<0.05,{ }^{* *} \mathrm{P}<0.01,{ }^{* * *} \mathrm{P}<0.001$. NFIB, nuclear factor I/B; siRNA, small interfering RNA; NC, negative control; ZO-1, zona occludens-1; EMT, epithelial-mesenchymal transition. 
A
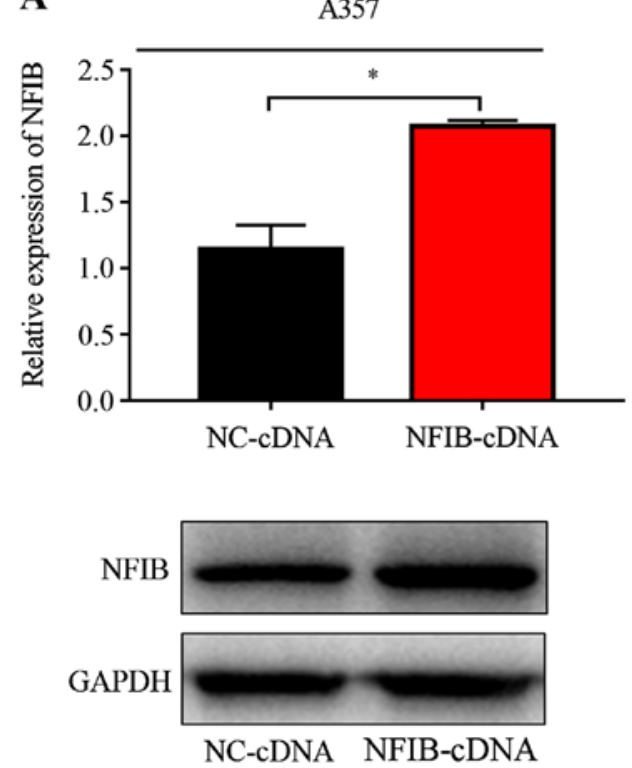

B
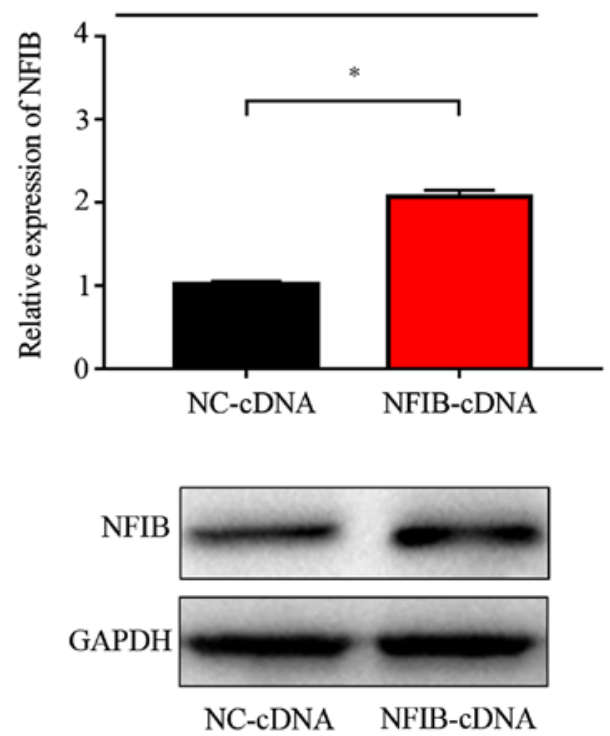

Figure 8. Detection of NFIB overexpression efficiency in melanoma cells. Reverse transcription-quantitative PCR and western blot analysis of (A) A375 and (B) A875 cell lines following NFIB-cDNA or NC-cDNA transfection. " $\mathrm{P}<0.05$. NFIB, nuclear factor I/B; NC, negative control.
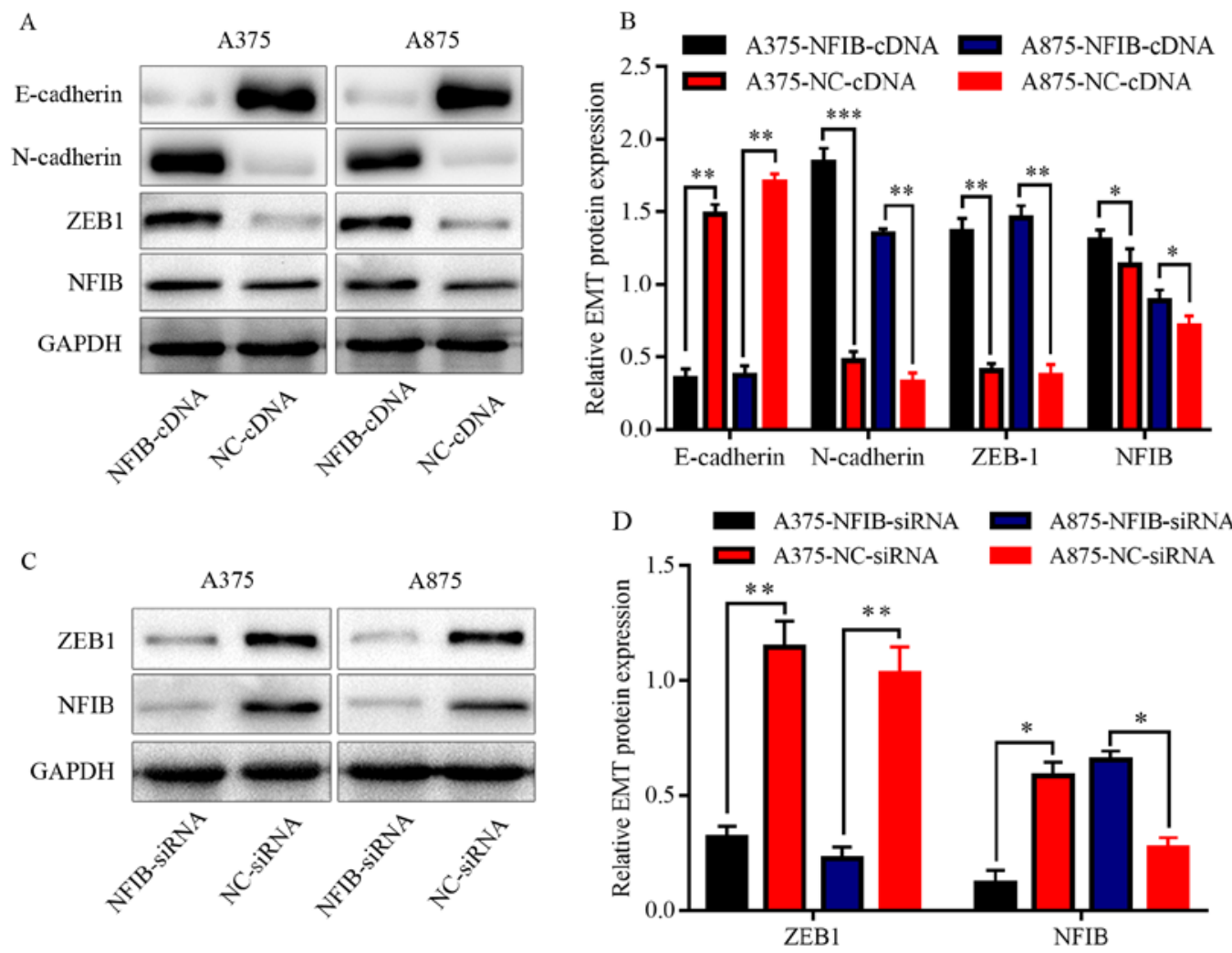

Figure 9. Western blot analysis results of epithelial-mesenchymal transition proteins in A375 and A875 cell lines. (A) Western blot and (B) semi-quantitative analysis of E-cadherin, N-cadherin, ZEB1 and NFIB following transfection with NIB-cDNA or NC-cDNA. (C) Western blot and (D) semi-quantitative analysis of ZEB1 and NFIB expression following transfection with NFIB-siRNA or NC-siRNA. ${ }^{*} \mathrm{P}<0.05,{ }^{* *} \mathrm{P}<0.01{ }^{* * *} \mathrm{P}<0.001$. NFIB, nuclear factor I/B; siRNA, small interfering RNA; NC, negative control; ZEB1, zinc finger protein E-box binding homeobox-1.

melanoma cells were observed under TGF- $\beta 1$ treatment and NFIB-siRNA transfection. During inverted phase-contrast microscopy, A375 and A875 cells that initially had epithelial morphology developed an elongated fibroblast-like morphology upon exposure to TGF- $\beta 1$-induced EMT. In contrast, the NFIB-siRNA group melanoma cells were closely packed, and the number of cells in the same field of view was higher, compared with the NC-siRNA group, therefore that TGF- $\beta 1$ treated NFIB knockdown cells mostly retained their primary epithelial morphology (Fig. 7A). Western blotting 
suggested that the downregulation of NFIB was accompanied by relatively higher expression levels of E-cadherin and ZO-1, compared with the control groups. Conversely, relatively low expression levels of $\mathrm{N}$-cadherin and VIM were associated with the NFIB-siRNA transfected cells (Fig. 7B and C). Overall, these data indicated that NFIB can promote EMT in melanoma cells.

NFIB positively regulates EMT by modulating ZEB1 in melanoma cell lines. To further elucidate the specific molecular mechanism of NFIB in the regulation of EMT in melanoma cells, the A357 and A875 cell lines were transfected with NFIB-cDNA groups or NC-cDNA. The overexpression was detected by RT-qPCR and western blot analysis. NFIB expression was overexpressed in A375 and A875 cells using transfection with NFIB-cDNA compared with NC-cDNA (Fig. 8).

In gastrointestinal epithelial tumors, it has been documented that NFIB is closely related to tumor EMT and promotes the occurrence of EMT in tumor cells by upregulating the expression of SNAI1. It was speculated that NFIB imbued its functions in melanoma by modulating its downstream signaling molecules. ZEB1 has been studied in various tumors and served an important role in EMT. However, the association between NFIB and ZEB1 remains unclear; thus, the present study proceeded to explore this in melanoma. In line with the data obtained from melanoma cells in western blotting, NFIB overexpression was associated with elevated ZEBland N-cadherin, while the expression of E-cadherin was downregulated (Fig. 9A and B). Supporting this, NFIB silencing downregulated ZEB1 in A375 and A875 cells, compared with NC-siRNA (Fig. 9C and D), indicating that ZEB1 was associated with NFIB in the regulation of EMT in melanoma.

\section{Discussion}

NFIB is a member of the NFI nuclear transcription factor family. NFIB plays an important role in the development of normal embryos and the formation and development of various organs by participating in DNA replication and transcription and can be detected and localized in the nucleus at the early stages of murine embryonic development at 14.5 days $(27,28)$. Accumulating studies indicate that NFIB is closely related to the occurrence of malignant tumors through gene fusion, especially in adenoid cystic carcinoma (29). However, some scholars have pointed out that NFIB may have tumor-suppressive effects as rearrangement leads to NFIB gene truncation and loss of function, together with other related genes, such as NFIB-AIG1, NFIB-MAN1A1 and NFIB-NKAIN2 (30-32). Due to this contradictory, double-sided effect of NFIB, which is characterized by both oncogenic and tumor-suppressive activity, the role of NFIB in melanoma remains unclear.

The present study demonstrated that NFIB was upregulated in human melanoma samples, relative to nevus and normal skin samples. Furthermore, NFIB expression was associated with a poor prognosis in melanoma. These are consistent with previous observational data in patients with non-small-cell lung cancer (31). Moreover, the present study revealed that high expression of NFIB was associated with poor prognosis in patients with melanoma. Consistent with the aforementioned NSCLC study, NFIB is overexpressed and regulates cell viability and proliferation during transformation of murine SCLC, where NFIB amplification is $\sim 15 \%$ of primary human SCLC (33).

Based on this evidence, and to further understand the biological functions of NFIB in melanoma cell lines, siRNA-mediated silencing of NFIB was employed to explore the role of this molecule. NFIB silencing inhibited melanoma cell proliferation, colony formation, as well as cell migration and invasion. The cell cycle of melanoma cells was analyzed; however, the experimental results were not satisfactory, and there is no suggestion that there was an association between NFIB expression and the cell cycle in melanoma (data not shown). However, in another study, NFIB knockdown in TP53-mutated triple-negative breast cancer cells promoted cell death, triggered cell cycle arrest and enhanced sensitivity to docetaxel, a first-line chemotherapeutic drug in breast cancer treatment (34). These results suggest that NFIB might serve an important role in melanoma.

A growing number of studies have proposed the behavior of EMT to be a reversible biological process that can regulate migration and invasion in human melanoma $(11,35,36)$. To examine the underlying biological functions by which NFIB promotes migration and invasion in melanoma cells, functional experiments were performed, through which it was uncovered that NFIB had a positive effect on the occurrence of EMT. A recent study revealed that NFIB was involved in the EMT process in colorectal cancer by encompassing various downstream effector molecules $(22,37)$. Western blots were not conducted on the groups treated with TGF- $\beta 1$, therefore further experimentation is needed to analyze this. Considering that NFIB regulates numerous cancer-related pathways, the downstream signaling pathway is of great significance for NFIB in the development of melanoma.

ZEB1 is a well-characterized transcription factor that facilitates tumor invasion and metastasis through an EMT-independent mechanism in carcinoma cells (12,38-40). Since ZEB1 belongs to the ZEB family and binds the E-box sequence CACCT, which has two possible binding sites for NFIB, finding a connection between NFIB would be useful to understand the oncogenic functions of NIFB in melanoma (41). Thus, it was hypothesized that NFIB might regulate ZEB1 to influence the EMT of melanoma. The present data support the idea that NFIB overexpression can increase ZEB1 expression. Conversely, the silencing of NFIB could repress ZEB1. However, the present conclusion only provides a new site for NFIB to regulate the biological process in melanoma; further extensive research is needed to verify this hypothesis and determine whether there is an NFIB-ZEB1 axis that modulates the process of EMT. Besides, additional in vivo, cell cycle and apoptosis assay experiments are worth conducting to determine whether NFIB contributes to other aspects of melanoma malignancy or if there exist ZEB2-related or other signaling pathways that are also linked with the function of NFIB in melanoma. Collectively, the present findings may hold promising insights into NFIB as a novel prognostic biomarker to predict prognosis and potential therapeutic target in melanoma. 


\section{Acknowledgements}

Not applicable.

\section{Funding}

The present study was supported by The National Nature Science Foundation of China (grant no. 81472000).

\section{Availability of data and materials}

The datasets used during the present study are available from the corresponding author upon reasonable request.

\section{Authors' contributions}

$\mathrm{RC}$ analyzed and interpreted the patient data. RC and SG performed the western blotting and functional experiments, and were major contributors in writing the manuscript. WH performed the statistical analysis of the experimental data and proofread the manuscript. YL collected the clinical data. YC contributed to the conception of the study and designed the research plan. All authors read and approved the final manuscript.

\section{Ethics approval and consent to participate}

The present study was approved by The Ethics Review Committee at Tongji Hospital affiliated with Huazhong University of Science and Technology. All participants signed an informed consent form.

\section{Patient consent for publication}

Not applicable.

\section{Competing interests}

The authors declare that they have no competing interests.

\section{References}

1. Siegel R, Naishadham D and Jemal A: Cancer statistics, 2012. CA Cancer J Clin 62: 10-29, 2012.

2. Siegel R, DeSantis C, Virgo K, Stein K, Mariotto A, Smith T, Cooper D, Gansler T, Lerro C, Fedewa S, et al: Cancer treatment and survivorship statistics, 2012. CA Cancer J Clin 62: 220-241, 2012.

3. Boyle GM: Therapy for metastatic melanoma: An overview and update. Expert Rev Anticancer Ther 11: 725-737, 2011.

4. Siegel R, Ma J, Zou Z and Jemal A: Cancer statistics, 2014. CA Cancer J Clin 64: 9-29, 2014.

5. Miller AJ and Mihm MC Jr: Melanoma. N Engl J Med 355: 51-65, 2006.

6. Mirzaei H, Gholamin S, Shahidsales S, Sahebkar A, Jaafari MR Mirzaei HR, Hassanian SM and Avan A: MicroRNAs as potential diagnostic and prognostic biomarkers in melanoma. Eur J Cancer 53: 25-32, 2016.

7. Eggermont AM and Kirkwood JM: Re-evaluating the role of dacarbazine in metastatic melanoma: What have we learned in 30 years? Eur J Cancer 40: 1825-1836, 2004.

8. Kalluri R and Weinberg RA: The basics of epithelial-mesenchymal transition. J Clin Invest 119: 1420-1428, 2009.

9. Pastushenko I and Blanpain C: EMT transition states during tumor progression and metastasis. Trends Cell Biol 29: 212-226, 2019.
10. Hanahan D and Weinberg RA: Hallmarks of cancer: The next generation. Cell 144: 646-674, 2011.

11. Caramel J, Papadogeorgakis E, Hill L, Browne GJ, Richard G, Wierinckx A, Saldanha G, Osborne J, Hutchinson P, Tse G, et al: A switch in the expression of embryonic EMT-inducers drives the development of malignant melanoma. Cancer Cell 24: 466-480, 2013.

12. Denecker G, Vandamme N, Akay O, Koludrovic D, Taminau J, Lemeire K, Gheldof A, De Craene B, Van Gele M, Brochez L, et al: Identification of a ZEB2-MITF-ZEB1 transcriptional network that controls melanogenesis and melanoma progression. Cell Death Differ 21: 1250-1261, 2014.

13. Blomquist P, Belikov S and Wrange O: Increased nuclear factor 1 binding to its nucleosomal site mediated by sequence-dependent DNA structure. Nucleic Acids Res 27: 517-525, 1999.

14. Gronostajski RM: Roles of the NFI/CTF gene family in transcription and development. Gene 249: 31-45, 2000.

15. Chaudhry AZ, Lyons GE and Gronostajski RM: Expression patterns of the four nuclear factor I genes during mouse embryogenesis indicate a potential role in development. Dev Dyn 208: 313-325, 1997.

16. Li S and Rosen JM: Nuclear factor I and mammary gland factor (STAT5) play a critical role in regulating rat whey acidic protein gene expression in transgenic mice. Mol Cell Biol 15: 2063-2070, 1995.

17. Kane R, Finlay D, Lamb T and Martin F: Transcription factor NF 1 expression in involuting mammary gland. Adv Exp Med Biol 480: 117-122, 2000

18. Chen KS, Lim JWC, Richards LJ and Bunt J: The convergent roles of the nuclear factor I transcription factors in development and cancer. Cancer Lett 410: 124-138, 2017.

19. Stringer BW, Bunt J, Day BW, Barry G, Jamieson PR, Ensbey KS, Bruce ZC, Goasdoué K, Vidal H, Charmsaz S, et al: Nuclear factor one B (NFIB) encodes a subtype-specific tumour suppressor in glioblastoma. Oncotarget 7: 29306-29320, 2016.

20. Moon HG, Hwang KT, Kim JA, Kim HS, Lee MJ, Jung EM, Ko E, Han W and Noh DY: NFIB is a potential target for estrogen receptor-negative breast cancers. Mol Oncol 5: 538-544, 2011.

21. Xing D, Bakhsh S, Melnyk N, Isacson C, Ho J, Huntsman DG, Gilks CB, Ronnett BM and Horlings HM: Frequent NFIB-associated gene rearrangement in adenoid cystic carcinoma of the vulva. Int J Gynecol Pathol 36: 289-293, 2017.

22. Liu Z, Chen J, Yuan W, Ruan H, Shu Y, Ji J, Wu L, Tang Q, Zhou Z, Zhang X, et al: Nuclear factor I/B promotes colorectal cancer cell proliferation, epithelial-mesenchymal transition and 5-fluorouracil resistance. Cancer Sci 110: 86-98, 2019.

23. Fane ME, Chhabra Y, Hollingsworth DEJ, Simmons JL, Spoerri L, Oh TG, Chauhan J, Chin T, Harris L, Harvey TJ, et al: NFIB Mediates BRN2 driven melanoma cell migration and invasion through regulation of EZH2 and MITF. Ebiomedicine 16: 63-75, 2017.

24. Hebert SL, Simmons C, Thompson AL, Zorc CS, Blalock EM and Kraner SD: Basic helix-loop-helix factors recruit nuclear factor I to enhance expression of the $\mathrm{NaV} 1.4 \mathrm{Na}^{+}$channel gene. Biochim Biophys Acta 1769: 649-658, 2007.

25. Livak KJ and Schmittgen TD: Analysis of relative gene expression data using real-time quantitative PCR and the 2(-Delta Delta C(T)) method. Methods 25: 402-408, 2001.

26. Chaffer CL, San Juan BP, Lim E and Weinberg RA: EMT, cell plasticity and metastasis. Cancer Metastasis Rev 35: 645-654, 2016.

27. Barry G, Piper M, Lindwall C, Moldrich R, Mason S, Little E, Sarkar A, Tole S, Gronostajski RM and Richards LJ: Specific glial populations regulate hippocampal morphogenesis. J Neurosci 28: 12328-12340, 2008.

28. Piper M, Barry G, Harvey TJ, McLeay R, Smith AG, Harris L, Mason S, Stringer BW, Day BW, Wray NR, et al: NFIB-mediated repression of the epigenetic factor Ezh2 regulates cortical development. J Neurosci 34: 2921-2930, 2014.

29. Becker-Santos DD, Lonergan KM, Gronostajski RM and Lam WL: Nuclear factor I/B: A master regulator of cell differentiation with paradoxical roles in cancer. EBioMedicine 22: 2-9, 2017.

30. Suzuki H, Aoki K, Chiba K, Sato Y, Shiozawa Y, Shiraishi Y, Shimamura T, Niida A, Motomura K, Ohka F, et al: Mutational landscape and clonal architecture in grade II and III gliomas. Nat Genet 47: 458-468, 2015.

31. Dooley AL, Winslow MM, Chiang DY, Banerji S, Stransky N, Dayton TL, Snyder EL, Senna S, Whittaker CA, Bronson RT, et al: Nuclear factor I/B is an oncogene in small cell lung cancer. Genes Dev 25: 1470-1475, 2011. 
32. Brayer KJ, Frerich CA, Kang H and Ness SA: Recurrent fusions in MYB and MYBL1 define a common, transcription factor-driven oncogenic pathway in salivary gland adenoid cystic carcinoma. Cancer Discov 6: 176-187, 2016.

33. Denny SK, Yang D, Chuang CH, Brady JJ, Lim JS, Grüner BM, Chiou SH, Schep AN, Baral J, Hamard C, et al: Nfib promotes metastasis through a widespread increase in chromatin accessibility. Cell 166: 328-342, 2016.

34. Liu RZ, Vo TM, Jain S, Choi WS, Garcia E, Monckton EA, Mackey JR and Godbout R: NFIB promotes cell survival by directly suppressing p21 transcription in TP53-mutated triple-negative breast cancer. J Pathol 247: 186-198, 2019.

35. Lin K, Baritaki S, Militello L, Malaponte G, Bevelacqua Y and Bonavida B: The role of B-RAF mutations in melanoma and the induction of EMT via dysregulation of the NF-KB/Snail/RKIP/PTEN circuit. Genes Cancer 1: 409-420, 2010.

36. Perrot CY, Javelaud D and Mauviel A: Insights into the transforming growth factor-beta signaling pathway in cutaneous melanoma. Ann Dermatol 25: 135-144, 2013

37. Wu C, Zhu X, Liu W, Ruan T, Wan W and Tao K: NFIB promotes cell growth, aggressiveness, metastasis and EMT of gastric cancer through the Akt/Stat3 signaling pathway. Oncol Rep 40: $1565-1573,2018$.
38. Ren J, Chen Y, Song H, Chen L and Wang R: Inhibition of ZEB1 reverses EMT and chemoresistance in docetaxel-resistant human lung adenocarcinoma cell line. J Cell Biochem 114: 1395-1403, 2013.

39. Zhang P, Sun Y and Ma L: ZEB1: At the crossroads of epithelial-mesenchymal transition, metastasis and therapy resistance. Cell Cycle 14: 481-487, 2015.

40. Spaderna S, Schmalhofer O, Wahlbuhl M, Dimmler A, Bauer K, Sultan A, Hlubek F, Jung A, Strand D, Eger A, et al: The transcriptional repressor ZEB1 promotes metastasis and loss of cell polarity in cancer. Cancer Res 68: 537-544, 2008.

41. Gheldof A, Hulpiau P, van Roy F, De Craene B and Berx G: Evolutionary functional analysis and molecular regulation of the ZEB transcription factors. Cell Mol Life Sci 69: 2527-2541, 2012.

\section{(i) $\ominus$}

This work is licensed under a Creative Commons Attribution-NonCommercial-NoDerivatives 4.0 International (CC BY-NC-ND 4.0) License. 